\title{
Support for a role of colour vision in mate choice in the nocturnal European treefrog (Hyla arborea)
}

\author{
Doris Gomez $^{1,3)}$, Christina Richardson ${ }^{2)}$, Thierry Lengagne ${ }^{2)}$, \\ Maxime Derex $^{2)}$, Sandrine Plenet ${ }^{2)}$, Pierre Joly ${ }^{2)}$, \\ Jean-Paul Léna ${ }^{2)} \&$ Marc Théry ${ }^{1)}$
}

( ${ }^{1}$ CNRS UMR 7179, Muséum National d'Histoire naturelle, Département d'Ecologie et de Gestion de la Biodiversité, 1 avenue du petit château, 91800 Brunoy, France; ${ }^{2}$ CNRS UMR 5023 Ecologie des Hydrosystèmes Fluviaux, Université Lyon 1, Université de Lyon, 43 Bd du 11 novembre 1918, 69622 Villeurbanne Cedex, France)

(Accepted: 15 September 2010)

\section{Summary}

Although nocturnal anurans use vision for reproductive communication, it remains unknown whether they see colours at night. Here, we explored this question in the European treefrog (Hyla arborea), by conducting two mate choice experiments under controlled light conditions. Experiments involved static male models with identical calls but different vocal sac colouration combining chromatic (red/orange) and brightness (dark/light) information. We found that females preferred dark red over light orange, evidencing for the first time a visually-guided mate choice in nocturnal diffuse light conditions. Conversely, females did not discriminate between dark orange and light red. The preference for dark over light in the first but not in the second experiment suggested that females had not only access to brightness cues but also to chromatic cues. The absence of preference may originate from females choosing at random in a situation where colour and brightness cues may convey contradictory information about male quality or from individual heterogeneity in the type of cues used for mate choice. Overall, these experiments provide the first support for the use of colour vision in a nocturnal amphibian.

Keywords: colour vision, brightness detection, rod, nocturnal anuran, mate choice.

3) Corresponding author's e-mail address: dgomez@mnhn.fr 


\section{Introduction}

Humans have a poor night vision; the role of vision in nocturnal communication has long been overlooked. Nocturnal anurans largely rely on acoustic signals for their communication (Gerhardt, 1994). Nevertheless, male visual displays — present in a large repertoire in numerous species (e.g., Amézquita \& Hödl, 2004) and often dimorphic in colouration (Hoffman \& Blouin, 2000) - have been recently shown to play a role in mate attraction, mate choice or competition (Rosenthal et al., 2004; Taylor et al., 2007; Vasquez \& Pfennig, 2007; Gomez et al., 2009).

Nocturnal anurans have a well-developed visual system, sensitive to very dim light conditions (Cummings et al., 2008) and presenting two rod classes (Liebman \& Entine, 1968). The possession of two rod classes may enable anurans to detect brightness (also called achromatic) or colour (also called chromatic) cues, depending on whether rod responses are summed or compared by the neural system. Although physiologically costly in terms of sensitivity, colour vision provides more reliable information than brightness and is as advantageous at night as during the day (Kelber \& Roth, 2006). To date, colour vision - the ability to discriminate two signals differing only in their spectral composition — has been demonstrated in only a few nocturnal species. In nocturnal anurans, colour vision has been evidenced in bright light (Hailman \& Jaeger, 1974) but visual ability at night remains completely unknown (Kelber \& Roth, 2006).

The only study testing the role of male vocal sac colouration in mate choice in a nocturnal anuran (Gomez et al., 2009) was conducted in the European treefrog (Hyla arborea). In this species, male vocal sac colouration contains carotenoids (Richardson et al., 2009), pigments which are traditionally viewed as costly to express; colour intensity likely reflects individual quality. A more intense colouration is more chromatic, that is more saturated in pigments (general rule for carotenoid-based colouration, review in Andersson \& Prager, 2006), and darker (specific case of vocal sac colouration in Hyla arborea; Gomez et al., unpublished results). Gomez et al. (2009) revealed that females preferred a more intense vocal sac colouration (darker and more chromatic, appearing dark red to a human eye) compared to a pale vocal sac colouration (lighter and less chromatic, appearing light orange to a human eye). Nevertheless, this study did not tell whether this preference was triggered by brightness and/or by colour (chromatic) cues. 
Here, night colour vision was tested in the European treefrog through mate choice experiments involving male models. Females were given the choice between dark red or light orange in a first experiment and between dark orange and light red in a second experiment. Based on the knowledge accumulated to date about the use of visual cues for mate choice in Hyla arborea (Gomez et al., 2009), we expect females to prefer dark red over light orange in the first experiment. This preference should translate into a preference for dark orange over light red if females predominantly use brightness cues, to light red over dark orange if females predominantly use colour cues, and to no preference if females use both available cues, the two latter patterns supporting a night colour vision.

\section{Methods}

\section{Female capture}

We investigated the existence of colour vision in a large metapopulation of $H$. arborea from the Isle Crémieu area, in Center-East France $\left(45^{\circ} 44^{\prime} 17^{\prime \prime} \mathrm{N}\right.$, $\left.5^{\circ} 21^{\prime} 07^{\prime \prime} \mathrm{E}\right)$. Females were captured at night using white head lights. Females in amplexus were tested during the night of their capture. Females found alone were kept in an outdoor enclosure at the pond along with males. Females were then either captured if found in amplexus or released after a few days.

\section{Protocol for testing colour vision in the context of mate choice}

We chose the European treefrog because it was the only anuran species for which male colouration has been explored for its biochemical bases (Richardson et al., 2009) and some of its possible functions (Gomez et al., 2009). The protocol designed to test the frogs' nocturnal vision did not follow a classic scheme for several reasons. It was dictated by observations made from the experiments conducted the year before (Gomez et al., 2009) which revealed important restrictions intrinsic to the biological system chosen. First, female arrival at the pond was highly irregular and unpredictable, inducing a chronic shortage in females. Second, female were only receptive for a few hours before they released their eggs. Third, experiments on vision required to ensure a dark adaptation that further reduced the time-window of female availability for experiments. Fourth, the proportion of females not 
responding to experimental conditions was substantial (see below) and we observed a general loss of interest of individuals after several trials (D.G. and C.R., pers. obs.). This loss of interest probably due to the use of non living males was already perceptible with videos and was anticipated to be even more problematic with the use of motionless models. A classic protocol (e.g., grey card experiment as mentioned by Kelber et al., 2003) based on a high number of animals/trials would have been unrealistic given the aforementioned constraints. We, thus, reduced testing to two experiments, a protocol which, although less powerful, was still informative enough to constitute the best compromise.

In each experiment, females were given the choice between two 1:1 scale resin models representing European treefrog males with inflated vocal sac and calling antiphonally. Models were preferred to living males because we could control in detail the acoustic and visual stimuli provided to females. The soundtrack was based on recordings from males of the local population and modified to construct synthetic calls identical for both male models. Model colouration was adjusted to match the frogs' visual perception (see below). For the back, we chose the average colouration found in the study population. Vocal sac colouration varied according to the experiment (Figure 1).

- Experiment 1 (exp. 1) opposed a male model with a light orange vocal sac to a male model with a dark red vocal sac (illustration of models in Appendix A, Figure A1).

- Experiment 2 (exp. 2) opposed a male model with a light red vocal sac to a male model with a dark orange vocal sac.

The terms 'red' and 'orange' referred to how humans perceive the vocal sac colouration of the males in the study population. Frogs likely do not perceive these hues as so. An increasing carotenoid pigmentation translates into an increase of colour saturation (increase of chroma) and a darkening. Although not specifically demonstrated for anurans, the relationship between pigment concentration and colour saturation appears to be the general rule for colours containing carotenoids (review in Hill \& McGraw, 2006; example in Navarro et al., 2010). The negative correlation between saturation and brightness has been found for males in different populations of Hyla arborea (Gomez et al., unpublished results). Finally, although rapid physiological colour change is widespread in anurans (Hoffman \& Blouin, 2000), it has never been observed for vocal sac colouration. In the genus Hyla, it has 
only been reported to concern dorsal and flank colouration (Buchanan, 1994; King et al., 1994). For all these reasons, it is reasonable to believe that an intense vocal sac colouration (dark and more chromatic) likely reflects a good-quality male.

The side on which we presented the red model was randomly chosen for a given experiment. There was no side bias in the stimulus presentation over the whole set of females tested (exp. 1: $N=42, \chi^{2}=3.4, p>0.05$; exp. $\left.2 N=39, \chi^{2}=0.03, p>0.05\right)$. There was no side bias in female choice (exp. 1: $N=20, \chi^{2}=0.8, p>0.05$; exp. $2: N=20, \chi^{2}=0.2$, $p>0.05)$, confirming that we assessed female preference for a male and not for a location. For a given experiment, all females were tested for the same single set of two models. The great care given to designing and building the models guarantees the good quality of the results even if all potential pseudoreplication problems may not have been ruled out. All statistics were performed using R 2.11.1 (2008).

\section{Experimental design}

Experiments took place in an indoor enclosure to control for ambient noise, light and weather conditions. The arena consisted of a $1 \mathrm{~m}$ equilateral triangle, the two male models occupying two apexes while the tested female was released at the third apex. We placed a loudspeaker (Monacor SP7 connected to a computer via a Stageline 102 amplifier) behind each model and defined a 'choice area' of $15 \mathrm{~cm}$ radius around it, where females could sit and watch the models. To improve sound quality and decrease sound reflection, we clothed the arena with foam pieces and covered the floor with a cork layer. We entirely covered the foam with wire netting to prevent females from entering foam cavities and the floor with a removable gunny rinsed in pond water between any two trials. We checked that the wire did not affect sound quality. We suspended a white LED light (Nichia NSPW510BS) above the arena in a way that the full arena received equal lighting; we adjusted its overall intensity over 350-700 $\mathrm{nm}$ to mimic dim moonlight or starlight conditions $\left(0.004 \mathrm{~cd} / \mathrm{m}^{2}\right)$, light conditions found under the vegetation cover during clear full moon nights or in open areas during overcast or new moon nights. In the European treefrog for which the occurrence of mating activities is closely related to rainfall (Blankenhorn, 1972), such light conditions are frequently encountered in nature. Finally, the frogs' movements were recorded in infrared light (Sony HDR-SR7E), a range of wavelengths to which frogs are blind (Jaeger \& Hailman, 1973). 


\section{Trial procedure}

Trials were performed between 2200 and $0500 \mathrm{~h}$. Each female took part in two trials, i.e., the two experiments, in a random sequence. Before its first trial, we placed the female for at least $1 \mathrm{~h}$ in a light-safe container ensuring sufficient dark adaptation. We handled the animals with a dim red headlight to ensure a minimal perturbation of the dark-adapted state (lack of rod sensitivity in this wavelength range; Figure 1). We randomly selected one female and placed her for $2 \mathrm{~min}$ in a restraining wire cage positioned one meter from both models. After this habituation period, we removed the cage lid, allowing the female to move freely for $20 \mathrm{~min}$. We considered that the female had made her choice when she remained for more than $20 \mathrm{~s}$ in one choice area. The choice was not valid if the female had not sampled both visual stimuli available (from the restraining cage or while in movement), stood motionless for 5 min after habituation, left the arena or remained less than $20 \mathrm{~s}$ in the choice areas.

Contrary to other previous mate choice experiments in frogs (Rosenthal et al., 2004; Taylor et al., 2007), we did not choose a criterion of first-visit to define female choice. Such a criterion would have introduced a potential first-visit bias by excluding females paying a close attention to visual stimuli before choosing their mate. Such behaviour was indeed observed in previous experiments for a substantial proportion of females which did not assess the stimuli from their restraining cage but only once released. These females paid short visits (which lasted for a few seconds) to both stimuli before choosing their mate (Gomez et al., 2009). It was also observed in the present experiments (see below). Such a close visual inspection may help females to decide and is likely adaptive for visual stimuli which, contrary to acoustic stimuli, are only perceptible over short distances.

After its first trial, the female was placed back in its light-safe container for at least half an hour before its second trial. Females showing a lack of receptivity - leaving the arena or staying motionless - were kept until the following night to be tested again before being released at the capture site. Females retained for testing were housed individually in boxes at a temperature of $6-8^{\circ} \mathrm{C}$ to delay oviposition. No female was kept for more than two consecutive nights. A total of 40 females was tested in the experiments (total of 81 trials). Out of these 14 made no valid choice and 26 made at least one valid choice. Twelve made a valid choice in one experiment only (6 in exp. 1 and 6 in exp. 2) and 14 made a valid choice in both experiments. 
As a consequence, there was a total of 20 females (40 trials) with a valid choice for each experiment and the results presented hereafter concern these females. It is noteworthy that $35 \%$ of all receptive females did not respond at all to the experiments and only half of the trials conducted were successfull.

\section{Stimuli preparation}

Several males from the Isle Crémieu metapopulation captured for other purposes had been audio recorded while calling. Using Avisoft SAS-lab pro software, we modified the call characteristics of a natural bout to reflect a high-quality male (Richardson et al., 2009) and compensate for the potentially poor attractiveness of static models: dominant frequency $2310 \mathrm{~Hz}$, amplitude $85 \mathrm{~dB}$ at $1 \mathrm{~m}$, bout duration $3.4 \mathrm{~s}$ and call rate 7.35 calls/s, corresponding to the lower decile (for dominant frequency), the mean (for call amplitude) and the upper decile (for bout duration and call rate) values for the study population. We constructed sound sequences $\left(3^{\prime \prime} 4^{\prime \prime \prime}\right.$ call- $1^{\prime \prime} 5^{\prime \prime \prime}$ silence) so that both models presented strictly identical and antiphonal calls with no overlap, a property known to affect male attractiveness in Hyla arborea (Richardson et al., 2008). We checked that both loudspeakers delivered the same sound amplitude before each trial by using a sound meter (Lutron SL-4001).

We adjusted model colouration to create a perceptual match for the frogs' vision between 'natural' and 'model' stimuli. This procedure, recommended and implemented for videos (Fleishman et al., 1998; Gomez et al., 2009), has never been applied to models but is crucial if models are to be involved in vision experiments. Any two visual stimuli are perceived as identical if they elicit the same neural outputs from the different classes of photoreceptors in the animal's eye (Fleishman et al. 1998). We, therefore, had to find the paint (of reflectance $R_{\text {paint }}$ ) that would satisfy the equations:

$$
\left\{\begin{array}{l}
\int R_{\text {male }} I_{\text {moonlight }} S_{\text {Rod S }} \mathrm{d} \lambda=\int R_{\text {paint }} I_{\text {LEDlight }} S_{\text {Rod S }} \mathrm{d} \lambda, \\
\int R_{\text {male }} I_{\text {moonlight }} S_{\text {Rod L }} \mathrm{d} \lambda=\int R_{\text {paint }} I_{\text {LEDlight }} S_{\text {Rod L }} \mathrm{d} \lambda,
\end{array}\right.
$$

where $I_{\text {moonlight }}$ is the moonlight irradiance spectrum digitized from Warrant (2004) using WinDig (Lovy, 1996), $I_{\text {LEDlight }}$ the white led light irradiance spectrum measured with a spectrometer (Avantes AvaSpec-3648-SPU2), calibrated (using an Avalight DH-CAL light source) and connected through a 


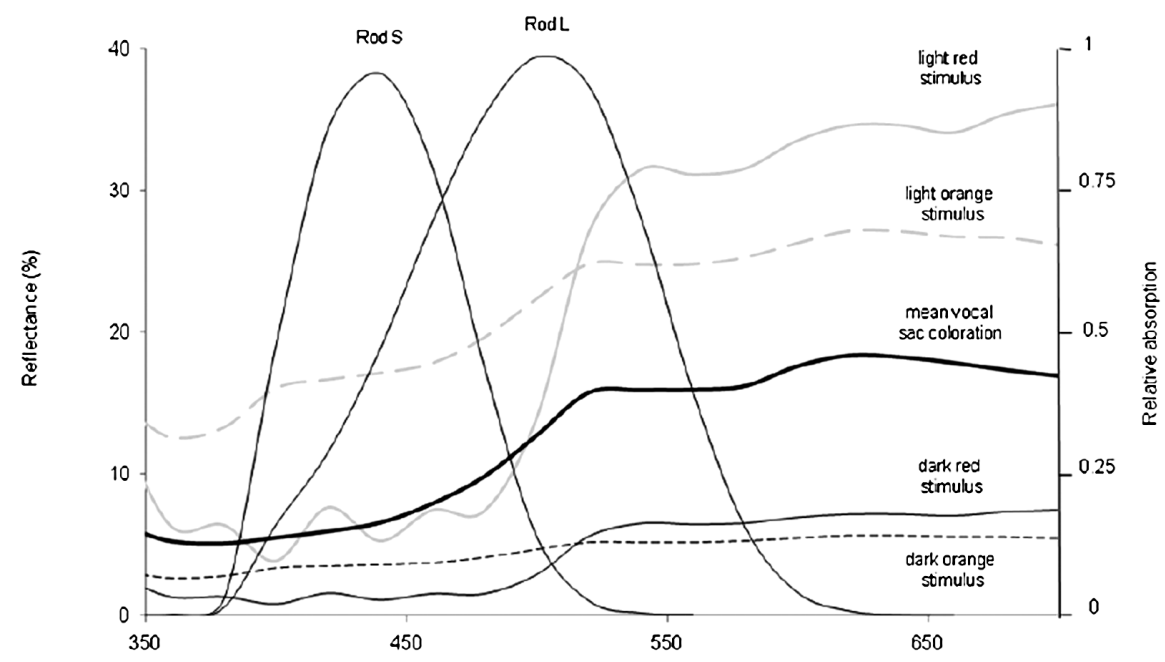

Wavelength $(\mathrm{nm})$

Figure 1. Reflectance spectra of the vocal sac stimuli used in the experiments: light red (grey plain line), light orange (grey dashed line), mean colouration in the study population (bold plain line), dark red (thin plain line), and dark orange (thin dashed line). Spectral sensitivity functions for rod sensitive to short (S) and long (L) wavelengths, used in vision modelling.

fibre (FC-UV600-2-ME) to a cosine-corrected sensor (Ocean Optics CC3$U V)$. We built the absorption functions of the rods ( $S_{\text {Rod S }}$ and $S_{\text {Rod L }}$; Figure 1) as in Gomez et al. (2009). For rod absorption peaks, we chose the data (435 nm and $503 \mathrm{~nm}$ ) obtained by King et al. (1993) for Hyla cinerea, a closely-related species. The location in wavelength of rod sensitivity is highly conserved among amphibians (430-435 nm for Rod S and 500$503 \mathrm{~nm}$ for Rod L in all adult amphibian species investigated to date; King et al., 1993 and references therein), making Hyla cinerea a good surrogate for the vision of its close relative. We used Govardovskii's widely recognised templates for vertebrate A1 pigments (Govardovskii et al., 2000) and data on lens transmission established for the Northern leopard frog Rana pipiens, the only nocturnal anuran species for which this data was available (Kennedy \& Milkman, 1956). Given the knowledge acquired to date on anuran vision, this model was the best approximation one could make of Hyla arborea's potential nocturnal vision.

First, we built the visual stimuli to test (of reflectance $R_{\text {male }}$ ) by using the natural range of colouration variation in the study population (see ex- 
ample of natural colouration in Appendix, Figure A2). A total of 125 males had been captured from the Isle Crémieu metapopulation during a capture campaign two years before the experiment (Gomez et al., 2009) and their colouration had been measured in spectrometry with a spectrometer (Avantes AvaSpec-3648-SPU2), a deuterium-halogen light source (Avantes AvaLightDHS) and a coaxial optic fibre (Avantes FCR-7UV200-2-45-ME) reflective to white reference and dark noise. We analysed the spectra using AVICOL (Gomez, 2010) and extracted the mean $m$ and standard deviation $\sigma$ of brightness (mean reflectance over the range 350-700 nm), chroma (difference between minimal and maximal reflectance over the mean reflectance over 350$700 \mathrm{~nm}$ ) and hue (maximal slope over the spectra), parameters commonly used to characterise spectral shape (e.g., Doutrelant et al., 2008). We built the stimuli by combining brightness (dark: mean - SD for brightness; light: mean + SD for brightness) and colour cues (red: mean + SD for hue and chroma; orange: mean - SD for hue and chroma). Light stimuli had similar brightness (quantum catch (in $\mu \mathrm{mol} / \mathrm{s}$ ) for $\operatorname{Rod} \mathrm{S}=0.055 \mu \mathrm{mol} / \mathrm{m}^{2}$ per s and for $\operatorname{Rod} \mathrm{L}=0.21 \mu \mathrm{mol} / \mathrm{m}^{2}$ per s; Appendix and Table A1). Dark stimuli had similar brightness (quantum catch for $\operatorname{Rod} S=0.025$; $\operatorname{Rod} L=0.095$; Appendix and Table A1).

Following this first computation step, we determined model colouration using acrylic paints (Dalbe fine Prismo paints: Yellow 116, Magenta 256, Cyan 400, White 118, Black 530). We mixed primary colour paints, applied the mixture on resin and varnished it (Dalbe matt water varnish), measured it in reflectance spectrometry using the equipment described above, and calculated rod quantum catches using equation (1) with the paint reflectance spectrum. We repeated this procedure until the equations could be satisfied and obtained all the stimuli used in experiments 1 and 2 (see Appendix for details, Table A1 and Figure A1).

\section{Results}

Females significantly preferred the dark red over the light orange vocal sac (exp. 1: $N=20$ (15 dark red/5 light orange), $\chi^{2}=5, p=0.025$, Figure 2) but showed no discrimination between light red and dark orange vocal sacs (exp. $2: N=20$ (11 light red/9 dark orange), $\chi^{2}=0.2, p=0.655$, Figure 2). Among the 14 females that had both trials validated, nine chose the 


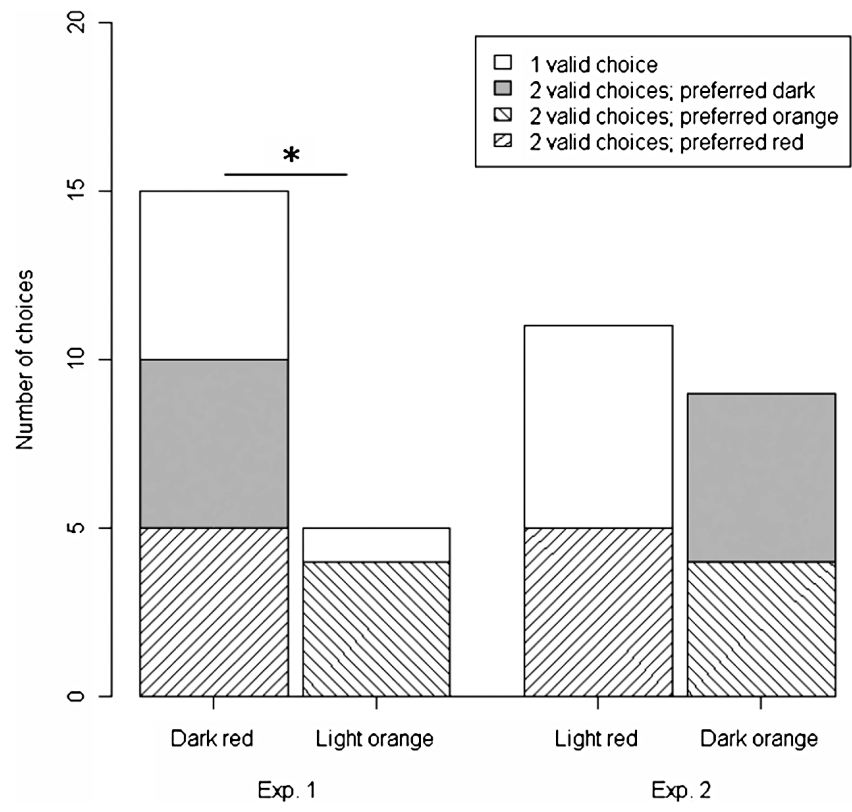

Figure 2. Female preference for vocal sac colouration presented by male models. Females responded to one (white) or two experiments (other fill). Among the latter, a preference was recorded in the two experiments for the dark stimulus (grey), the red stimulus (dashed $45^{\circ}$ ), the orange stimulus $\left(\right.$ dashed $\left.-45^{\circ}\right)$. * Significant difference $(p<0.05)$.

same hue in both experiments ( 5 for the red, 4 for the orange, Figure 2) while five chose the same brightness in both experiments ( 5 for the dark, 0 for the light, Figure 2). However, there was no significant preference for chromatic cues $\left(N=14\right.$ (9 hue/5 brightness), $\left.\chi^{2}=1.14, p=0.286\right)$. Receptive females chose quickly in both experiments ( $4^{\prime} 21^{\prime \prime}$ in exp. 1, $4^{\prime} 20^{\prime \prime}$ in exp. 2$)$ and the length of time they took to make their choice was similar whatever the model chosen (Kruskal-Wallis tests, exp. 1: $\chi^{2}=0.069, p=0.792$; exp. 2: $\left.\chi^{2}=2.668, p=0.102\right)$. While the majority of females chose the male model that they first visited (90\% in exp. 1, 70\% in exp. 2) without visiting the alternative male model (75\% in exp. 1, 60\% in exp. 2), a non-negligible proportion of females visited both models (25\% in exp. 1, $40 \%$ in exp. 2). A large proportion of females gave the model a close visual inspection, standing within 1-2 cm of the model (55\% of all females in exp. 1, 45\% of all females in exp. 2), sometimes including a tactile inspection as for amplexus solicitation ( $25 \%$ of all females in exp. 1, $15 \%$ of all females in exp. 2). 


\section{Discussion}

In dim-light conditions, females preferred a dark red over a light orange male model, which confirmed the results previously obtained with video playbacks (Gomez et al., 2009). By using models which overcome the limitations (absence of 3D cues, horizontal gradient of light intensity) imposed by videos, we showed for the first time that female treefrogs used male colour signals to choose their partner under nocturnal conditions.

The preference for the dark red vocal sac was not followed by any consistent preference for a specific vocal sac colouration once colour and brightness cues were dissociated, a result inconsistent with an exclusive use of brightness or colour cues for mate choice. Female preference for a dark red over a light orange colouration is robust (models, this study; videos, Gomez et al., 2009). Moreover, we adjusted model colouration so that dark stimuli had the same perceived brightness and we did the same in the case of light stimuli. The difference between the two stimuli of the same brightness level $(<0.01-0.03 \mu \mathrm{mol} / \mathrm{s})$ was much lower than the difference between the light and dark stimuli opposed in one experiment $(0.11-0.13 \mu \mathrm{mol} / \mathrm{s}$; Table A1). Hence, if females only used brightness cues to choose their partner, they should have shown a significant preference for the dark orange in exp. 2 . Similarly, if frogs had disregarded any brightness information, they should have shown a significant preference for the red stimulus, here the light red, matching the preference pattern previously expressed with models or videos.

The absence of general preference in exp. 2 may originate from two nonexclusive mechanisms. First, females may have chosen their partner at random in a situation where stimuli potentially conveyed contradictory information about investment in colouration and individual quality. Under that hypothesis, a general preference would be observed only if colour and brightness redundantly signal a high-quality (dark red) or a low-quality (light orange) male. Second, females may differ in the way they assess male attractiveness. Some may assess brightness cues while others may assess chromatic cues before choosing a mate. Under that hypothesis, the heterogeneity at an individual level would be masked when colour and brightness are redundant signals of mate quality (exp. 1) but revealed when colour and brightness cues potentially convey conflicting information about mate quality (exp. 2). Yet, the 'unnatural' and conflicting aspect of vocal sac colouration only exists for individuals able to process chromatic and brightness information and would not exist for colour-blind individuals. 
These results are the first element of response to the long-asked but nevertested question of the existence of colour vision in nocturnal amphibians. This support is still fragile because we chose to depart from classic vision tests based on conditioning and/or on a high number of tests and animals/trials (Kelber et al., 2003). We built an original but less powerful protocol adapted to the constraints imposed by the biological system - species reluctant to conditioning (Kelber et al., 2003), limited female availability and responsiveness, specific context of mate choice. We are confident in the results, as ensured by the thorough preparation of visual stimuli and the robustness of female preference. We hope these results will increase research attention on this question.

So far, night colour vision has been shown in a few species (Kelber \& Roth, 2006), all invertebrate species but one, the gecko (Roth \& Kelber, 2004), a reptile with a pure cone retina. Amphibians are the only vertebrates to possess two types of rod (Liebman \& Entine, 1968). Contrary to most vertebrates which sacrifice colour vision at night to increase light sensitivity, amphibians may have a dichromatic night colour vision. Anatomical (large eyes) and neural (summation of photoreceptor outputs Warrant, 1999) adaptations may help them to improve the reliability of visual information at night. Our results suggest females exploit not only chromatic but also brightness cues. This can be an efficient strategy to decrypt a visually complex scene (Schaefer et al., 2006), locate small or moving targets (Osorio et al., 1999) and interpret redundant signals of quality. Overall, it may help females to choose their mate rapidly under natural conditions, that is under the risk of predation.

\section{Acknowledgements}

We warmly thank Louis de Torhout for his precious help with creating frog models, Françoise Viénot for light intensity measurements, Romain Durieux for his important contribution to field work, and the mayors of Optevoz and Annoisin-Chatelans for giving us access to the field sites. This study was conducted in accordance with French laws and with the approval of the Préfecture de l'Isère (decision 2007-03328) and the Direction of Veterinary Services (permit DSV no. 69266347). It was supported by an ANR grant (COLAPSE programme).

\section{References}

Amézquita, A. \& Hödl, W. (2004). How, when, and where to perform visual displays: the case of the Amazonian frog Hyla parviceps. - Herpetologica 60: 420-429. 
Andersson, S. \& Prager, M. (2006). Quantifying colors. — In: Bird coloration, Vol. 1. Mechanisms and measurements (Hill, G.E. \& McGraw, K.J., eds). Harvard University Press, Cambridge, MA, p. 41-89.

Blankenhorn, H.J. (1972). Meteorological variables affecting onset and duration of calling in Hyla arborea L. and Bufo calamita calamita Laur. - Oecologia 9: 223-234.

Bowmaker, J.K. (2008). Evolution of vertebrate visual pigments. — Vision Res. 46: 20222041.

Buchanan, B.W. (1994). Sexual dimorphism in Hyla squirella - chromatic and pattern variation between the sexes. - Copeia: 797-802.

Cummings, M.E., Bernal, X.E., Reynaga, R., Rand, A.S. \& Ryan, M.J. (2008). Visual sensitivity to a conspicuous male cue varies by reproductive state in Physalaemus pustulosus females. - J. Exp. Biol. 211: 1203-1210.

Doutrelant, C., Grégoire, A., Grnac, N., Gomez, D., Lambrechts, M.M. \& Perret, P. (2008). Female coloration indicates female reproductive capacity in blue tits. - J. Evol. Biol. 21: 226-233.

Fleishman, L.J., McClintock, W.J., D’eath, R.B., Brainard, D.H. \& Endler, J.A. (1998). Colour perception and the use of video playback experiments in animal behaviour. Anim. Behav. 56: 1035-1040.

Gerhardt, H.C. (1994). The evolution of vocalization in frogs and toads. - Annu. Rev. Ecol. Syst. 25: 293-324.

Gomez, D. (2010). AVICOL v4. A program to analyse spectrometric data. - Free program available from the author upon request at dodogomez@yahoo.fr or by download from http://sites.google.com/site/avicolprogram/

Gomez, D., Richardson, C., Lengagne, T., Plenet, S., Joly, P., Léna, J.-P. \& Théry, M. (2009). The role of nocturnal vision in mate choice: females prefer conspicuous males in the European treefrog (Hyla arborea). - Proc. Roy. Soc. Lond. B: Biol. 276: 2351-2358.

Govardovskii, V.I., Fyhrquist, N., Reuter, T., Kuzmin, D.G. \& Donner, K. (2000). In search of the visual pigment template. - Vis. Neurosci. 17: 509-528.

Hailman, J.P. \& Jaeger, R.G. (1974). Phototactic responses to spectrally dominant stimuli and use of color vision by adult anuran amphibians - comparative survey. - Anim. Behav. 22: 757-795.

Hill, G.E. \& McGraw, K.J. (2006). Bird coloration. - Harvard University Press, Cambridge, MA.

Hoffman, E.A. \& Blouin, M.S. (2000). A review of colour and pattern polymorphisms in anurans. - Biol. J. Linn. Soc. 70: 633-665.

Jaeger, R.G. \& Hailman, J.P. (1973). Effects of intensity on the phototactic responses of adult anuran amphibians: a comparative survey. — Z. Tierspsychol. 33: 352-407.

Kelber, A. \& Roth, L.S.V. (2006). Nocturnal colour vision - not as rare as we might think. - J. Exp. Biol. 209: 781-788.

Kelber, A., Vorobyev, M. \& Osorio, D. (2003). Animal colour vision — behavioural tests and physiological concepts. - Biol. Rev. 78: 81-118.

Kennedy, D. \& Milkman, R.D. (1956). Selective light absorption by the lenses of lower vertebrates, and its influence on spectral sensitivity. — Biol. Bull. 111: 375-386.

King, R.B., Douglass, J.K., Phillips, J.B. \& Baube, C.L. (1993). Scotopic spectral sensitivity of the optomotor response in the green treefrog Hyla cinerea. - J. Exp. Zool. 267: 40-46. 
King, R.B., Hauff, S. \& Phillips, J.B. (1994). Physiological color-change in the green treefrog - responses to background brightness and temperature. - Copeia: 422-432.

Liebman, P. \& Entine, G. (1968). Visual pigments of frog and tadpole (Rana pipiens). Vision Res. 8: 761-775.

Lovy, D. (1996). WinDig 2.5. — Freeware available from the author at dominique.lovy@ chiphy.unige.ch or by download from http://www.unige.ch/sciences/chifi/epb/winding. html (verified May 2004), Department of Physical Chemistry, University of Geneva, Geneva.

Navarro, C., Perez-Contreras, T., Aviles, J.M., McGraw, K.J. \& Soler, J.J. (2010). Beak colour reflects circulating carotenoid and vitamin A levels in spotless starlings (Sturnus unicolor). - Behav. Ecol. Sociobiol. 64: 1057-1067.

Osorio, D., Vorobyev, M. \& Jones, C.D. (1999). Colour vision of domestic chicks. — J. Exp. Biol. 202: 2951-2959.

R Development Core Team (2008). R: a language and environment for statistical computing. - R Foundation for Statistical Computing, Vienna. Available online at http://www.Rproject.org

Richardson, C., Léna, J.-P., Joly, P. \& Lengagne, T. (2008). Are leaders good mates? A study of call timing and male quality. - Anim. Behav. 76: 1487-1495.

Richardson, C., Popovici, J., Bellvert, F. \& Lengagne, T. (2009). Conspicuous colouration of the vocal sac of a nocturnal chorusing treefrog: carotenoid-based? - Amphibia-Reptilia 30: 576-580.

Rosenthal, G.G., Rand, A.S. \& Ryan, M.J. (2004). The vocal sac as a visual cue in anuran communication: an experimental analysis using video playback. - Anim. Behav. 68: $55-58$.

Roth, L.S.V. \& Kelber, A. (2004). Nocturnal colour vision in geckos. — Proc. Roy. Soc. Lond. B: Biol. 271: S485-S487.

Schaefer, H.M., Levey, D.J., Schaefer, V. \& Avery, M.L. (2006). The role of chromatic and achromatic signals for fruit detection by birds. - Behav. Ecol. 17: 784-789.

Taylor, R.C., Buchanan, B.W. \& Doherty, J.L. (2007). Sexual selection in the squirrel treefrog Hyla squirella: the role of multimodal cue assessment in female choice. - Anim. Behav. 74: 1753-1763.

Vasquez, T. \& Pfennig, K.S. (2007). Looking on the bright side: females prefer coloration indicative of male size and condition in the sexually dichromatic spadefoot toad, Scaphiopus couchii. - Behav. Ecol. Sociobiol. 62: 127-135.

Vorobyev, M. \& Osorio, D. (1998). Receptor noise as a determinant of colour thresholds. Proc. Roy. Soc. Lond. B: Biol. 265: 351-358.

Warrant, E.J. (1999). Seeing better at night: life style, eye design and the optimum strategy of spatial and temporal summation. - Vis. Res. 39: 1611-1630.

Wyszecki, G. \& Stiles, W.S. (1982). Color science, concepts and methods, quantitative data and formulae. - Wiley, New York, NY.

\section{Appendix A}

\section{Construction of visual stimuli}

In Vorobyev and Osorio's model of colour vision (Vorobyev \& Osorio, 1998), two colours $A$ and $k A$ ( $k A$ being values of $A$ multiplied by $k$ at each wavelength) generate no colour contrast 
Table A1. Vocal sac colouration in models and live males.

\begin{tabular}{llcccc}
\hline & Vocal sac colouration & $Q_{\text {Rod S }}$ & $Q_{\text {Rod L }}$ & $Q_{\text {Rod L }} / Q_{\text {Rod S }}$ & $Q_{\text {Rod S }}+Q_{\text {Rod L }}$ \\
\hline Experiment 1 & Light orange & 0.07 & 0.21 & 3.2 & 0.28 \\
& Dark red & 0.02 & 0.11 & 5.2 & 0.13 \\
Experiment 2 & Dark orange & 0.03 & 0.08 & 3.2 & 0.11 \\
& Light red & 0.04 & 0.21 & 5.6 & 0.25 \\
Natural colours & Mean + SD & 0.07 & 0.24 & 3.4 & 0.31 \\
& Mean - SD & 0.02 & 0.10 & 5.2 & 0.12 \\
\hline
\end{tabular}

All quantum catches are expressed in $\mu \mathrm{mol} / \mathrm{s}$. $Q_{\text {Rod S }}$ and $Q_{\text {Rod L }}$ describe the quantum catches of the rods sensitive to short and long wavelengths respectively. The $Q_{\operatorname{Rod~L}} / Q_{\text {Rod S }}$ ratio describes colour intensity; a colour for which a photoreceptor is more excited relative to the others is perceived as a more intense and more chromatic and pure colour (Wyszecki \& Stiles, 1982). The terms 'red' and 'orange' designate intensely and poorly saturated colours, respectively. Light colours describe colours for which quantum catches are high (here $>0.25$ for $Q_{\text {Rod L }}$ ), while dark colours describe colours for which quantum catches are low (here $<0.13$ for $Q_{\text {Rod L }}$ ).

if the ratio of the quantum catches of the different photoreceptors is identical for $A$ and for $k A$. As a consequence, we set the paints of the same hue to show similar ratio of quantum catches $Q_{\text {Rod L }} / Q_{\text {Rod S }}$ in order that these colours offer the same chromatic information. Orange colours had a ratio of 3.2 while red colours had a ratio of approx. 5.4 (Table A1).

Nothing is known about brightness detection in nocturnal anurans but some hypotheses have been put forward. Brightness detection can be based on the sum of the responses of the two types of rod (Kelber \& Roth, 2006). Alternatively, because rods sensitive to longer wavelengths represent $95 \%$ of the whole rod population (Bowmaker, 2008), they can alone
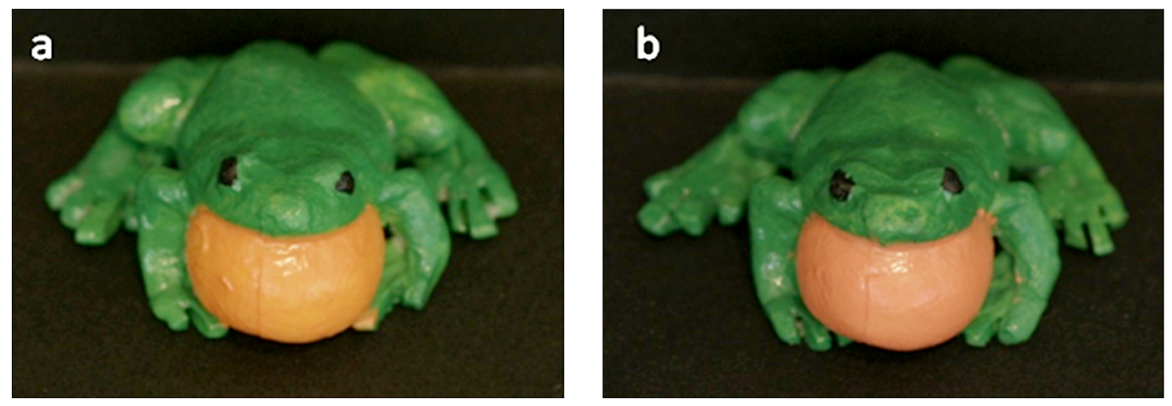

Figure A1. Dark red (a) and light orange (b) 1:1 scale resin models used in experiment 1. Green colouration reproduces the visual impression given by the mean back colouration of males in the study population. 'Red' and 'orange' refer to how humans perceive the natural vocal sac colours in males but not the paints of the models. OMaxime Derex. This figure is published in colour in the online edition of this journal, which can be accessed via 


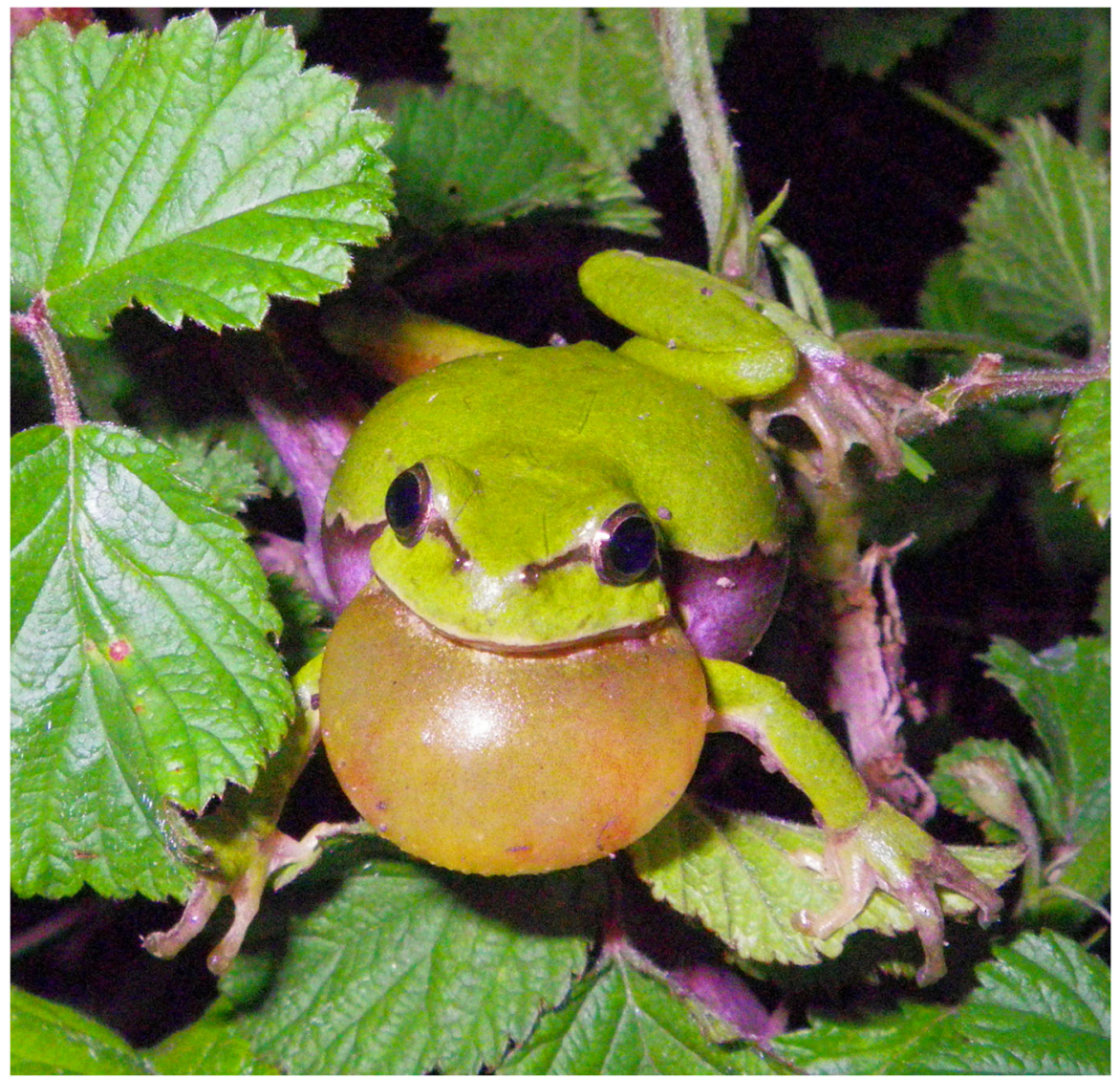

Figure A2. Adult male calling at night nearby the pond and displaying its vocal sac colouration. (Mathieu Troïanowski. This figure is published in colour in the online edition of this journal, which can be accessed via http://www.brill.nl/beh

contribute to brightness detection. To account for these two possible mechanisms, we checked that the paints of the same brightness had similar $Q_{\text {Rod L }}$ and similar $Q_{\text {Rod S }}+Q_{\text {Rod L }}$. Light colours had a sum of approx. 0.26 while dark colours offered twice less brightness with a value at 0.12 (Table A1). The ratio and sum of rod outputs were not exactly the same (Table A1) for two reasons. First, it was impossible to exactly match the colours for the two possible brightness detection mechanisms. Second, the visual effect of a paint mixture could not be predicted but was obtained empirically and we chose the best compromise. 


\title{
Support for a role of colour vision in mate choice in the nocturnal European treefrog (Hyla arborea)
}

\author{
Doris Gomez $^{1,3)}$, Christina Richardson ${ }^{2)}$, Thierry Lengagne ${ }^{2)}$, \\ Maxime Derex ${ }^{2)}$, Sandrine Plenet ${ }^{2)}$, Pierre Joly ${ }^{2)}$, \\ Jean-Paul Léna ${ }^{2)}$ \& Marc Théry ${ }^{1)}$
}

( ${ }^{1}$ CNRS UMR 7179, Muséum National d'Histoire naturelle, Département d'Ecologie et de Gestion de la Biodiversité, 1 avenue du petit château, 91800 Brunoy, France; ${ }^{2}$ CNRS UMR 5023 Ecologie des Hydrosystèmes Fluviaux, Université Lyon 1, Université de Lyon, 43 Bd du 11 novembre 1918, 69622 Villeurbanne Cedex, France)

(Accepted: 15 September 2010)

In the above-mentioned article, published in Behaviour 147/13-14 on pages $1753-1768$, on page 1759 the sentence:

Using Avisoft SAS-lab pro software, we modified the call characteristics of a natural bout to reflect a high-quality male (Richardson et al., 2009) and compensate for the potentially poor attractiveness of static models: dominant frequency $2310 \mathrm{~Hz}$, amplitude $85 \mathrm{~dB}$ at $1 \mathrm{~m}$, bout duration $3.4 \mathrm{~s}$ and call rate 7.35 calls/s, corresponding to the lower decile (for dominant frequency), the mean (for call amplitude) and the upper decile (for bout duration and call rate) values for the study population.

should read:

Using Avisoft SAS-lab pro software, we modified the call characteristics of a natural bout to reflect a high-quality male (Richardson et al., 2009) and compensate for the potentially poor attractiveness of static models: dominant frequency $2460 \mathrm{~Hz}$, amplitude $83 \mathrm{~dB}$ at $1 \mathrm{~m}$, bout duration 3.4 $\mathrm{s}$ and call rate 7.3 calls/s, corresponding to one standard deviation away from the mean values for the study population.

3) Corresponding author's e-mail address: dodogomez@yahoo.fr 
The authors would like to add that this change does not change anything to the results (as the soundtrack is the same throughout the whole experiment, for both potential targets presented) or experimental design of the study published in Behaviour. The authors apologise for any inconvenience caused. 